\title{
Doubling Silicon Ablation Process Efficiency and Improving Quality Using High Power High Repetition Rate Green Laser with TimeShift ${ }^{\mathrm{TM}}$ Capability
}

\author{
Rajesh PATEL ${ }^{* 1}$, James BOVATSEK ${ }^{* 1}$ and Hang-Ru GOY ${ }^{* 2}$ \\ ${ }^{*}$ Spectra Physics, 3635 Peterson Way, Santa Clara, CA 95054, USA \\ E-mail: raj.patel@spectra-physics.com \\ ${ }^{* 2}$ Hewlett Packard, 138 Depot Road, Singapore, Singapore 109683
}

\begin{abstract}
In an inkjet printer head a silicon barrier plate exists between an orifice plate and ink reservoir. This silicon plate needs to be machined to create slot to let ink through to the nozzle. While currently available green Diode Pumped Solid State (DPSS) lasers have done adequate job in machining silicon for creating slot, a new generation of high-power, high-repetition rate green Quasar ${ }^{\circledR}$ hybrid fiber laser with TimeShift ${ }^{\mathrm{TM}}$ capability developed by Spectra Physics allows doubling of silicon ablation process efficiency. By optimizing power, repetition rate, pulse width, number of pulses in a burst, and pulse separation time between sub-pulses within a burst, we have demonstrated that material removal efficiency can be doubled. We have also demonstrated that quality of the ablated features can be improved tremendously. We present experimental results of careful and systematic study that shows huge benefit higher power and repetition rate along with complete temporal control of pulse output can provide for silicon ablation.
\end{abstract}

DOI: 10.2961/jlmn.2015.02.0005

Keywords: Inkjet print head, Silicon, Ablation, Green Laser, burst machining, Quasar

\section{Introduction}

When inkjet printers were first ushered into the printing landscape in the 1980s, its low cost of ownership and superior quality proposition to the consumer destined its inexorable displacement of the rudimentary and noisy dot matrix incumbent. Over the next 3 decades, propelled by exploding demand, inkjet technology has scaled rapidly with ever increasing quality and speed, keeping up with the pace of consumer's changing needs and sophistication. Today, annual combined worldwide revenue of inkjet cartridges sale stands at a staggering $\$ 9.9$ billion [1].

Along the rapidly changing technological upward trajectory, we witnessed the fiction-to-reality of photo quality printing on a home printer. This was enabled by, among other things, continuous miniaturization of devices on the print head. In the case of thermal inkjet technology, one that encompasses complex thin film circuits, hordes of densely arranged thermal heaters(that are responsible for firing picolitre ink drop through the nozzles) and hair-thin, micro-fluidic or ink channels onto a single tiny Silicon (Si) die needed to be developed. The ink delivery through a slot presents the best technological enabler for high speed, and high resolution color printing, by virtue of the fact that two rows of nozzles can be closely sited, one on each side of the slot.

Ability to achieve a narrow slot width becomes very important, since it frees up precious real estate on the die for adding more devices and nozzles, as well as allowing a more compact layout for die and hence shrinking its size. This also helps keep the ink cartridges cost competitive. From a manufacturing perspective creating many through slots on one wafer is technologically demanding. This is further complicated by the fact that typically thickness of the wafer is $\sim 700 \mu \mathrm{m}$ and slot width can be as low as $100 \mu \mathrm{m}$. Figure 1 shows schematic cross sectional view of a typical monolithic inkjet printer head [2].

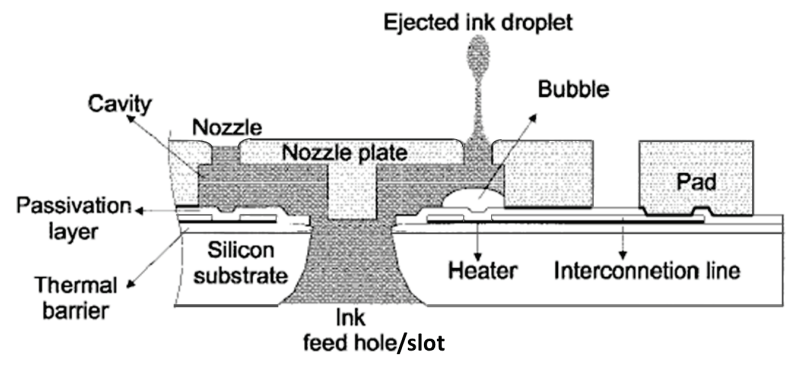

Fig. 1 Schematic cross sectional view of a typical monolithic inkjet printer head.

There are multiple ways to create ink slots on the Si wafer; but to do so in a high volume high quality low cost manufacturing environment, not many fit the bill. From a technical point of view, sandblasting is one of the early processes that were capable and adequate to do the job. However it has a process limitation on how narrow a slot can be cut. It is also not capable of handling new generation of product designs that have multifold increase in the number of slots to be cut on a single wafer. The process yield becomes low for a densely packed slot array design due to increased risk of cracking wafers. On top of that, it is also a high cost, high waste, high maintenance, and high operator intervention process.

Consequently very quickly laser cutting became an attractive way for creating slots in $\mathrm{Si}$, namely on 3 fronts $\mathrm{A}$ ) 
better manufacturing yield and quality, B) technological enabler for new product and C) cost of manufacturing. Precision laser cutting became a possibility for print head manufacturing with the advent of industrial grade ultraviolet (UV)/green Q-switched (Q-SW) Diode Pump Solid State (DPSS) lasers; it makes tenable Si micromachining with very small heat affected zone. That long-held quest to break the barrier of slot width could finally be realized.

But one of the challenges for laser process was it needed to do the job a lot faster. In its technological infancy, throughput for laser process was paltry and was a huge downside for print head manufacturers to fully adopting it to meet the ever expanding business and capacity needs. Obviously to meet throughput demand manufacturer can install a huge base of laser drilling machines, but it takes up premium shop floor real estate; that aside, it also requires a significant capital investment and making spare parts management and inventory a very challenging task.

Over the years, with rapid advancement in industrial laser power, laser cutting throughput has been able to scale up considerably from its early days. However it would not scale continuously in that regime given the constraint of die fragility that, among others, is dependent on the pulse energy and pulse-to-pulse overlap. Moderating pulse energy is traditionally accomplished by dialing up the pulse repetition frequency (PRF) of the higher power laser, while the pulse-to-pulse overlap is controlled by the scanning speed of the scan equipment. However, for current generation of Q-SW DPSS lasers continuously increasing PRF to achieve cutting goals is not possible since laser power drops very significantly as the PRF increases and pulse width and pulse-to-pulse energy variation increases significantly which will result in very inconsistent cutting results.

Hence, beyond the brute power and PRF increasing approach, novel methods of laser processing that ingeniously exploit, newly or yet to be discovered, efficient lasermaterial-time-space interaction would have to kick in. One such method that has shown some success in the lab and attracted much interest in the research community is the double pulse processing technique. Dual identical pulses, at similar pulse width and similar combined pulse energy to a typical single pulse, separated by a short temporal space in the same order as the pulse width, was shown to be able to increase many folds the percussion drilling speed of metals [3]. Thus, future lasers that are capable of delivering configurable pulse formats will predictably have a compelling play and value proposition for Si slot cutting process.

\section{Hybrid fiber laser}

While Q-SW DPSS lasers have fulfilled the demand from industry for creating small accurate features in a manufacturing environment, they do have limitations in achieving higher processing speeds and maintaining quality of features at higher speed. An intuitive approach to increasing the processing speed is to increase the laser's pulse repetition frequency (PRF) while holding other process parameters fixed. However, for a typical green or ultraviolet Q-SW DPSS laser, this is really not an option. For these lasers, the average power and pulse energy decrease quite rapidly as the PRF increases. Also, the laser pulse width and pulse-to-pulse energy variability tend to increase significantly. Since the variation in these laser parameters affects the micromachining speed, feature size, and accuracy achieved, simply increasing PRF is often not enough to maintain process results as one attempts to increase throughput. The real and desired solution to overcoming these limitations is a laser that not only maintains high average power at the higher PRFs but also continues to deliver constant pulse widths with low pulse-to-pulse energy variation.

Recognizing the need for such laser technology, at Spectra-Physics we have developed the Quasar ${ }^{\circledR}$ hybrid fiber laser (see Fig. 2). It is a laser with a unique combination of higher power at higher PRF. It also features TimeShift ${ }^{\mathrm{T}}$ technology which allows a wide range of independently software-settable pulse energies and pulse widths. In addition, TimeShift technology provides the ability to create sophisticated pulse-intensity waveforms in the time domain, through techniques such as pulse shaping, pulse splitting, and burst mode operation. With an output power of $>60 \mathrm{~W}$ at $355 \mathrm{~nm}$ wavelength at $300 \mathrm{kHz} \mathrm{PRF}$, and $>75 \mathrm{~W}$ at $532 \mathrm{~nm}$ wavelength at $200 \mathrm{kHz}$ PRF this laser offers very high power at high repetition rates. At the same time, in contrast to conventional DPSS Q-SW lasers it removes the dependence of pulse width on PRF, making it possible to truly hold all of the critical characteristics of the laser output steady as throughput is scaled up by increasing the PRF.

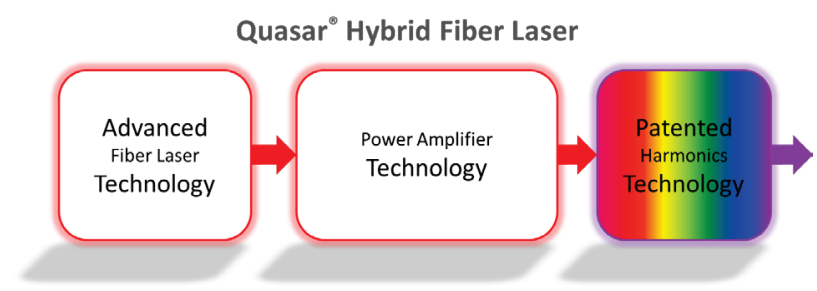

(a)

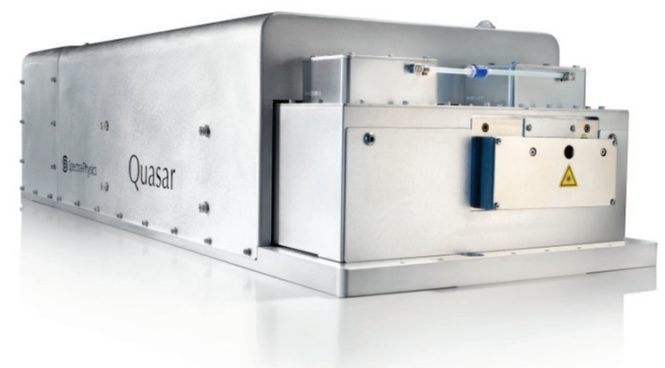

(b)

Fig. 2 Quasar hybrid fiber laser (a) schematic concept and (b) product.

\section{Experimental set up}

In this paper we have explored the benefit of, the combination of higher power at high PRF, independently adjustable pulse width, and the new advanced pulse manipulation capabilities for silicon ablation process using Quasar® 532nm laser.

\subsection{Laser system}

Spectra-Physics' Quasar ${ }^{\circledR}$ 532-75 hybrid fiber laser shown in Fig. 1 was used for this study. The laser is optimized for $200 \mathrm{kHz}$ performance and generates $>75 \mathrm{~W}$ for 
$375 \mu \mathrm{J}$ pulse energy output with short, 10ns pulse durations. The key specifications for this system are listed in Table 1.

Table 1. Key specifications for Spectra Physics' Quasar ${ }^{\circledR}$ 532-75 laser system.

\begin{tabular}{lc}
\hline Wavelength & $532 \mathrm{~nm}$ \\
Average Power & $\begin{array}{c}>75 \mathrm{~W} @ 200 \mathrm{kHz}, \\
10 \mathrm{~ns} \text { pulse width }\end{array}$ \\
$\begin{array}{l}\text { Max Pulse Energy } \\
\begin{array}{l}\text { Pulse Repetition Fre- } \\
\text { quency range }\end{array}\end{array}$ & $>375 \mu \mathrm{J}$ \\
$\begin{array}{l}\text { TimeShift } \\
\text { mable Program- }\end{array}$ & $<5$ to $>100 \mathrm{MHz}$ \\
$\mathrm{M}^{2}$ & $<1.3$ \\
Pulse-to-Pulse Stability & $<5 \% \mathrm{rms} 1 \sigma$ \\
\hline
\end{tabular}

\subsection{Optical set up}

The schematic of optical set up used for the experiments is shown in Fig. 3. It consists of a scanning galvanometer system (Scanlab Hurryscan II 14) with a $100 \mathrm{~mm}$ focal length telecentric $\mathrm{f}$-theta lens. The scan head is integrated into a precision linear motor XYZ positioning system (Newport Corporation IMS series stages and XPS motion controller) for full flexibility with regard to parts positioning and beam focusing capabilities. The Quasar ${ }^{\circledR}$ laser beam was expanded using beam expander to achieve beam diameter of $\sim 3.3 \mathrm{~mm}$ at the scan head aperture. This resulted in a round-beam focus diameter $\left(1 / \mathrm{e}^{2}\right)$ of $\sim 30 \mu \mathrm{m}$ at silicon wafer.

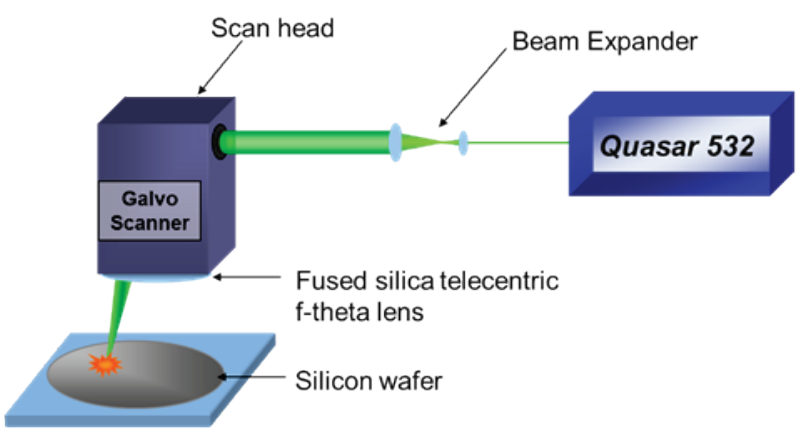

Fig. 3 Schematic of experimental set up.

\subsection{Ablation scan pattern}

Ablation trenches in the silicon were micro machined by scanning the laser beam in a serpentine raster pattern. Six adjacent lines, each separated by the raster spacing of $30 \mu \mathrm{m}$, comprise a single iteration of the scan routine. This scan routine was then repeated for $\mathrm{N}=5,10$, and 15 iterations, with the goal of generating trench depths in the range of $50-100 \mu \mathrm{m}$. Measuring depth of trenches in this range was more repeatable and consistent. Trenches deeper than $100 \mu \mathrm{m}$ in depth typically suffered from excessive debris clogging and were very difficult to measure accurately. Fig. 4 below illustrates the scan pattern and the example of resultant trench in the silicon wafer.

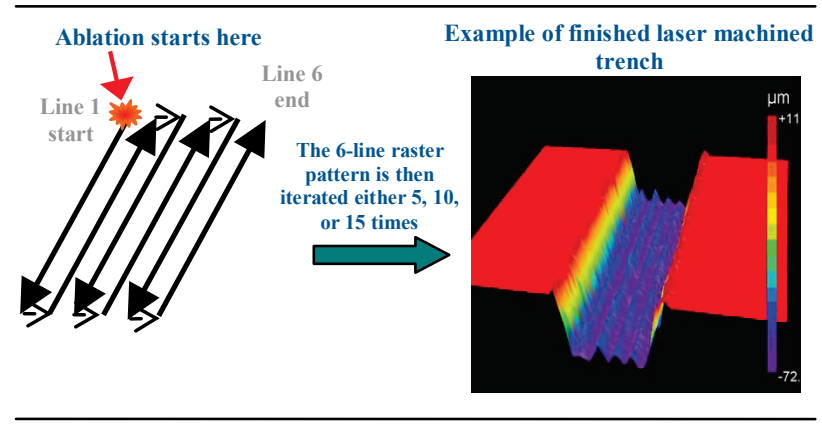

Fig. 4 Scan pattern for the trench and example of resulting trench in silicon.

\section{Results and discussion}

The baseline trench against which all the improvements achieved using Quasar ${ }^{\circledR}$ laser was created using a DPSS Q-SW green laser outputting a Gaussian pulses of $47 \mathrm{~ns}$ pulse width and $204 \mu \mathrm{j}$ per pulse energy at $135 \mathrm{kHz}$ PRF. A baseline trench depth of $73 \mu \mathrm{m}$ was achieved with $900 \mathrm{~mm} / \mathrm{s}$ scan speed, 6 raster lines, and with 5 raster iterations, which computes to a reference average scribing speed $V_{\text {ref }}$ of $30 \mathrm{~mm} / \mathrm{s}$. Since the Quasar ${ }^{\circledR}$ laser was operated at various PRF's and different scan speeds to optimize trench scribing process, the resulting trench depth and average speed were different than the reference case. So in order to determine the amount of depth increase, and hence improvement in ablation efficiency, a calculation for equivalent depth was required. Once the equivalent depth is known, a depth multiplier factor was calculated by dividing equivalent depth by baseline case depth. E.g. let's say a trench depth of $87 \mu \mathrm{m}$ was achieved using Quasar ${ }^{\circledR}$ at an average scribing speed of $48 \mathrm{~mm} / \mathrm{s}$ for a given set of laser parameters, then equivalent depth would be, $87 x(48 / 30)=$ $139.2 \mu \mathrm{m}$. Hence, the depth multiplier in this case would be $(139.2 / 73)=1.9$, which implies Quasar ${ }^{\circledR}$ laser can create same depth trench at a scribing speed of 1.9 times faster (almost twice as fast) than the baseline process case.

After a few preliminary ablation trials using Quasar ${ }^{\circledR}$ laser to roughly gauge silicon ablation behavior, we found that throughput and quality were changing with pulse width and different burst pulse waveforms This prompted a more focused study using three select pulse waveforms shown in Fig. 5 that were generated using TimeShift ${ }^{\mathrm{TM}}$ technology.

\subsection{Ablation depth improvement}

Trenches in silicon were created using the ablation scan pattern described above in section 3.3 for each of the waveform shown in Fig. 5. The depth of each trench was measured and depth multiplier factors were calculated and are shown in Table 2. 


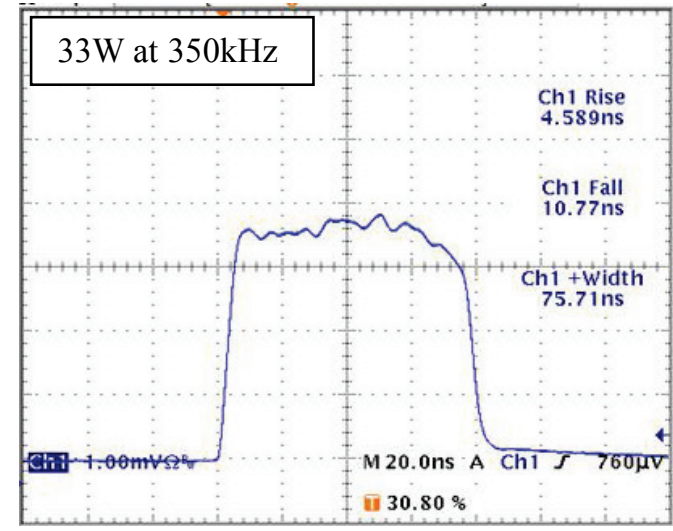

(a)Waveform 1: 60ns single pulse

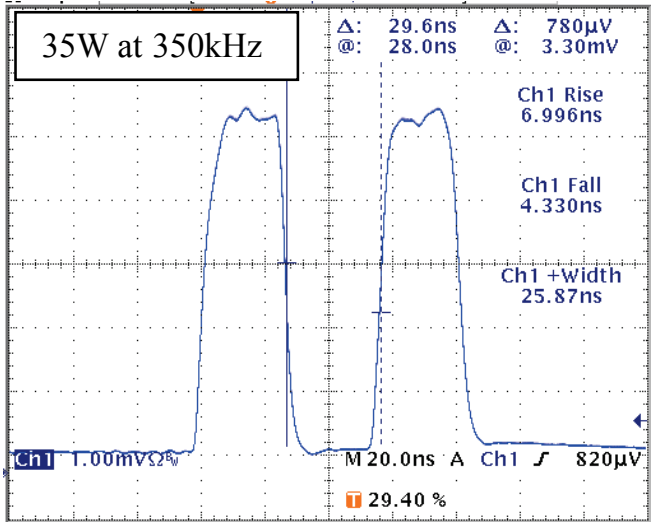

(b) Waveform 2: $2 \times 25 \mathrm{~ns}$ double pulse with $30 \mathrm{~ns}$ separation

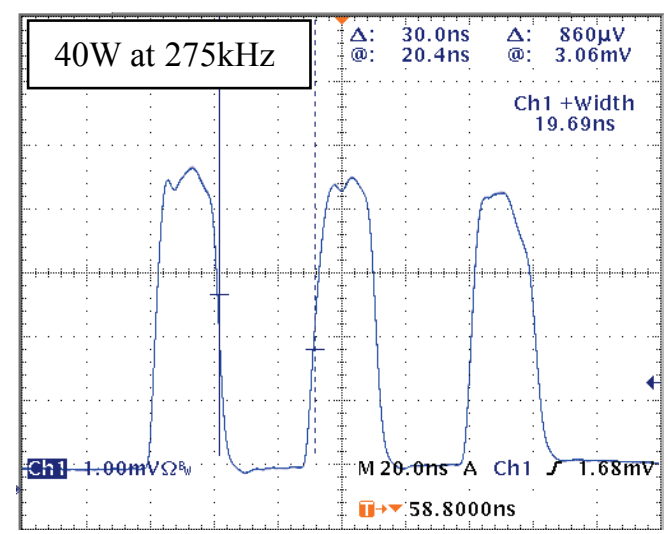

(c) Waveform 3: 3x20ns triple pulse with 30ns separation

Fig. 5 Three waveforms studied to improve throughput and quality of silicon ablation.
Table 2. Processing results for baseline case and three different waveforms.

\begin{tabular}{|c|c|c|c|c|c|}
\hline $\begin{array}{l}\text { Laser Process } \\
\text { Description }\end{array}$ & $\begin{array}{l}\text { Power on } \\
\text { Target (W) }\end{array}$ & $\begin{array}{c}\text { Pulse } \\
\text { Energy }(\mu)\end{array}$ & $\begin{array}{c}\text { Avg. Scribe } \\
\text { Speed } \\
(\mathrm{mm} / \mathrm{s}) \\
\end{array}$ & $\begin{array}{l}\text { Trench } \\
\text { Depth } \\
(\mu \mathrm{m})\end{array}$ & $\begin{array}{c}\text { Depth } \\
\text { Multiplier }\end{array}$ \\
\hline $\begin{array}{l}\text { Base line case - } \\
\text { Single } 47 \mathrm{~ns} \\
\text { pulse, } 135 \mathrm{kHz}\end{array}$ & 27.5 & 204 & 30 & 73 & 1.0 \\
\hline $\begin{array}{l}\text { Quasar } 532-75 \text {, } \\
\text { Single } 60 \mathrm{~ns} \\
\text { pulse, } 350 \mathrm{kHz}\end{array}$ & 33 & 94 & 48 & 87 & 1.9 \\
\hline $\begin{array}{l}\text { Quasar 532-75, } \\
2 \times 25 \mathrm{~ns}, 30 \mathrm{~ns} \\
\text { separation } \\
\text { double pulse, } \\
350 \mathrm{kHz}\end{array}$ & 35 & 100 & 48 & 107 & 2.3 \\
\hline $\begin{array}{l}\text { Quasar } 532-75 \text {, } \\
3 \times 20 \mathrm{~ns}, 30 \mathrm{~ns} \\
\text { separation } \\
\text { triple pulse, } \\
275 \mathrm{kHz}\end{array}$ & 40 & 145 & 69 & 85 & 2.7 \\
\hline
\end{tabular}

The data in Table 2 clearly shows that much deeper trenches can be created by optimizing Quasar ${ }^{\circledR}$ laser parameters and using proper TimeShift ${ }^{\mathrm{TM}}$ enabled waveform. With single $60 \mathrm{~ns}$ wide pulse at high PRF of $350 \mathrm{kHz}$ depth multiplier factor of 1.9 was observed, which indicates that we can create same depth trench at almost twice the speed compared to baseline case process speed. Further optimization of waveforms indicates using three 20ns wide pulses separated by $30 \mathrm{~ns}$ at $275 \mathrm{kHz}$ PRF waveform depth multiplier of 2.7 was possible which indicates that we can create same depth trench at almost three times the baseline process speed. While different trench depth was achieved for different process conditions, the trench width at top of the trench was $\sim 180 \mu \mathrm{m}$ for all trenches. The trench depth data clearly shows that using high power, high repetition rate, TimeShift $^{\mathrm{TM}}$ optimized waveforms provided by Quasar ${ }^{\circledR}$ laser can improve silicon ablation throughput by a factor of 2 to 3 .

\subsection{Quality improvement}

In addition to throughput improvement we were also interested in analyzing effect of various waveforms on quality of ablation. In Fig. 6 below, optical microscope images of the Quasar ${ }^{\circledR} 532-75$ single square pulse waveform and double pulse waveform processes are compared side-by-side with the baseline process. 

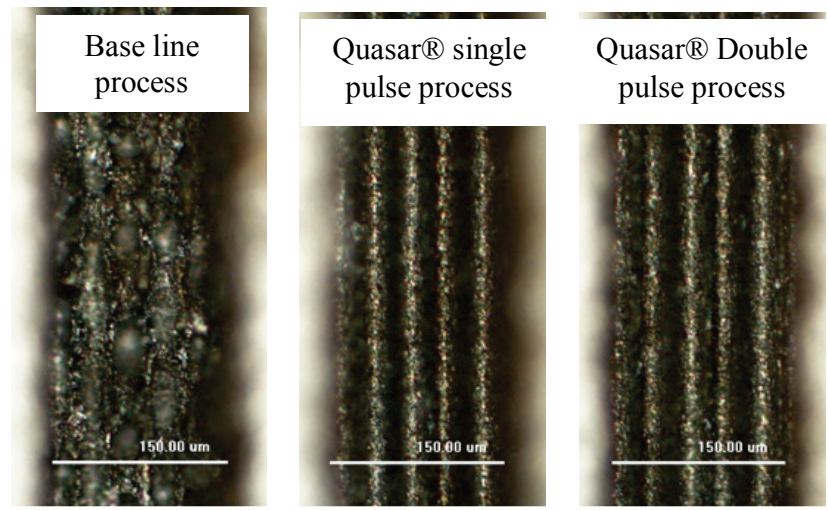

Fig. 6 Microscope picture of bottom of trenches.

In addition to microscope pictures shown in Fig. 6 we studied the quality of trenches by studying 3D images of trenches obtained using white light interferometer. Two such images of trench are shown in Fig. 7.

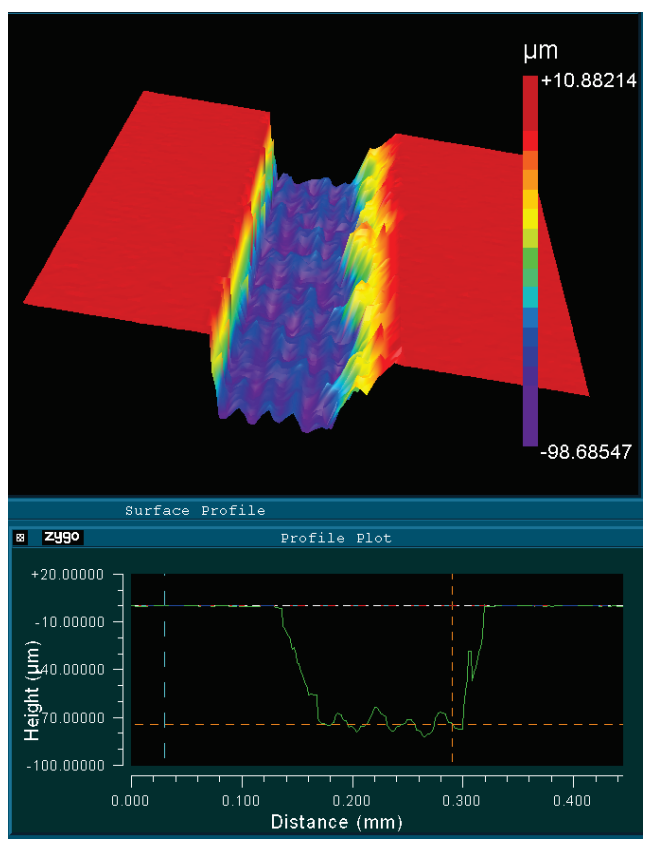

(a) Baseline process

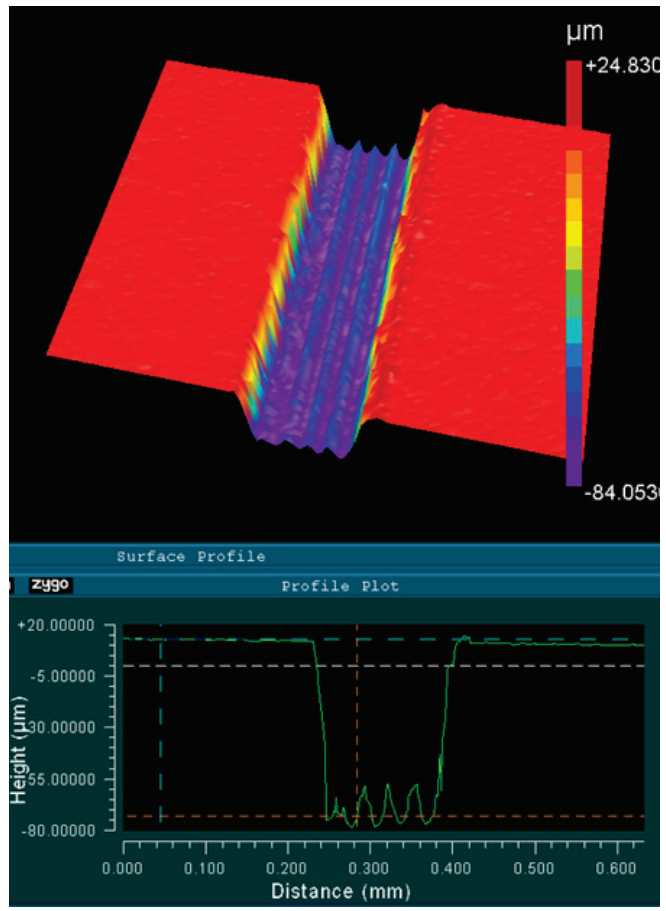

(b) Quasar® triple pulse process

Fig. 7 Example of 3D image of trench obtained using white light interferometer.

Both microscope images and 3D images of trenches shows cleaner trench with less re-solidified molten material at the bottom for trenches created using Quasar ${ }^{\circledR}$ laser. Generally speaking, the higher quality of the Quasar ${ }^{\circledR}$ cuts is likely attributable to two factors. One factor is very short rise time of the irradiating pulses from Quasar ${ }^{\circledR}$ laser. This rapid pulse rise time to full intensity means heat will not travel as far out of the focused beam spot before ablation is initiated, resulting in a lower volume of material that is heated, but not ablated or otherwise removed. Second factor is that in the case of double and triple pulse waveform ablation, the silicon is irradiated by smaller energy packets which, while energetic enough to cause significant volume ablation, they do not necessarily create too much excess heat that diffuses outside the ablation area.

\section{Conclusions}

A cost effective high throughput high quality slot drilling process in $\mathrm{Si}$ is very important for continued success of thermal inkjet technology. While Q-SW DPSS lasers have enabled the laser slot cutting process thus far, a new laser and process is necessary for supporting current and future need for creating higher density narrow slots on a $\mathrm{Si}$ wafer. Quasar ${ }^{\circledR}$ hybrid fiber laser is a unique laser with higher average power at higher PRF and a completely flexible laser pulse energy manipulation in time feature called TimeShift ${ }^{\mathrm{TM}}$. The experimental results show that, compared to an existing conventional Q-SW laser process, an increase in throughput by a factor of 2.7 with a simultaneous improvement in quality possible. Given the infinite possibility for customized pulse tailoring provided by Quasar ${ }^{\circledR}$ laser, it is quite possible that the speed of slot cutting process can be further increased. 


\section{References}

[1] Ink Jet/Laser Computer Printer Sales Statistics, Statis-
tic Brain, March 2, 2014,

http://www.statisticbrain.com/ink-jet-laser-computerprinter-sales-statistics/

[2] Jae-Duk Lee, Jun-Bo Yoon, Jae-Kwan Kim, Hoon-Ju Chung, Choon-Sup Lee, Hi-Deok Lee, Ho-Jun Lee, Choong-Ki Kim, Chul-Hi Han: "A Thermal Inkjet Printhead with a Monolithically Fabricated Nozzle Plate and Self-aligned Ink Feed Hole" J. of Microelectromechanical Systems, Vol. 8, No. 3, Sept. 1999, pg. 229-236.

[3] A.C. Forsman, P.S. Banks, M.D. Perry, E.M. Campbell, A.L. Dodell, and M.S. Armas: "Double-pulse machining as a technique for the enhancement of material removal rates in laser machining of metals" J. of Applied Physics, 98, 2005.

(Received: June 25, 2014, Accepted: January 30, 2015) 